\title{
Development of a multi-patient ventilator circuit with validation in an ARDS porcine model
}

\author{
Benjamin P. Wankum ${ }^{1} \cdot$ Riley E. Reynolds ${ }^{1}$ (D) Andrea R. McCain ${ }^{1} \cdot$ Nathaniel T. Zollinger $^{1} \cdot$ Keely L. Buesing $^{2}$. \\ Russel D. Sindelar ${ }^{3} \cdot$ Frank M. Freihaut $^{3} \cdot$ Tariku Fekadu $^{4} \cdot$ Benjamin S. Terry $^{1}$
}

Received: 21 December 2020 / Accepted: 22 May 2021 / Published online: 1 June 2021

(c) Japanese Society of Anesthesiologists 2021

\begin{abstract}
Purpose The COVID-19 pandemic threatens our current ICU capabilities nationwide. As the number of COVID-19 positive patients across the nation continues to increase, the need for options to address ventilator shortages is inevitable. Multi-patient ventilation (MPV), in which more than one patient can use a single ventilator base unit, has been proposed as a potential solution to this problem. To our knowledge, this option has been discussed but remains untested in live patients with differing severity of lung pathology.

Methods The objective of this study was to address ventilator shortages and patient stacking limitations by developing and validating a modified breathing circuit for two patients with differing lung compliances using simple, off-the-shelf components. A multi-patient ventilator circuit (MPVC) was simulated with a mathematical model and validated with four animal studies. Each animal study had two human-sized pigs: one healthy and one with lipopolysaccharide (LPS) induced ARDS. LPS was chosen because it lowers lung compliance similar to COVID-19. In a previous study, a control group of four pigs was given ARDS and placed on a single patient ventilation circuit (SPVC). The oxygenation of the MPVC ARDS animals was then compared to the oxygenation of the SPVC animals.

Results Based on the comparisons, similar oxygenation and morbidity rates were observed between the MPVC ARDS animals and the SPVC animals.

Conclusion As healthcare systems worldwide deal with inundated ICUs and hospitals from pandemics, they could potentially benefit from this approach by providing more patients with respiratory care.
\end{abstract}

Keywords COVID-19 $\cdot$ Coronavirus $\cdot$ Artificial respiration $\cdot$ Pandemics $\cdot$ Viruses

$\begin{array}{ll}\text { Abbreviations } \\ \text { ARDS } & \text { Acute Respiratory Distress Syndrome } \\ \text { FRE } & \text { Flow resistor } \\ \text { I-time } & \text { Inhalation time } \\ \text { LPS } & \text { Lipopolysaccharide }\end{array}$

Benjamin P. Wankum and Riley E. Reynolds contributed equally as primary author.

Riley E. Reynolds

riley.reynolds@huskers.unl.edu

1 Department of Mechanical and Materials Engineering, University of NE-Lincoln, Lincoln, NE 68588, USA

2 Department of General Surgery, University of Nebraska Medical Center, Omaha, NE 68198, USA

3 Department of Respiratory Care, The Nebraska Medical Center, Omaha, NE 68198, USA

4 Yakatit 12 Hospital Medical College, Addis Ababa, Ethiopia
MPV Multi patient ventilation

MPVC Multi patient ventilation circuit

PC Pressure control setting on the ventilator

PEEP Positive end expiration pressure

$\mathrm{PH} \quad$ Healthier patient

PS Sicker (ARDS) patient

SPVC Single patient ventilation circuit

\section{Introduction}

COVID-19 is a contagious respiratory disease caused by SARS-CoV-2 with varying degrees of respiratory illness from mild to life-threatening. Patients with a severe case of COVID-19 may develop acute respiratory distress syndrome (ARDS) caused by the lung injury sustained from viral infection [1,2]. Patients with severe ARDS oftentimes require invasive mechanical ventilation. In an observational 
study of 11,729 COVID-19 patients admitted into a hospital, $16.8 \%$ required mechanical ventilation [3]. The global spread of the novel coronavirus has caused concerns about overburdening the health care system with patients in respiratory failure. The continued increase in cases of COVID-19 may lead to a shortage of medical supplies and staff.

One proposed solution is patient stacking, where ventilator circuits are adapted to accommodate more than one patient at a time. This technique has been demonstrated a handful of times on a lung simulator, an animal model, and in humans [4-10]. Beitler et al. demonstrated how MPV can be performed on COVID-19 patients [10]. They included criteria for matching patients and concluded that identification of compatible patient pairs is essential to safely implementing MPV. However, COVID-19 patients with ARDS exhibit variable respiratory impedances and can be difficult to match, especially in smaller hospitals and clinics. Characteristics of ARDS include a decrease in lung compliance and an increase in airway resistance [11-14]. Additional characteristics of ARDS include bilateral infiltrates in the lungs, poor oxygenation, and acuity of onset [15]. The Berlin criteria were developed to categorize the degree of ARDS severity as determined by the partial pressure of arterial oxygen/fraction of inspired oxygen $\left(\mathrm{PaO}_{2} / \mathrm{FiO}_{2}\right)$. A ratio of $<200$ defines moderate ARDS, and $<100$ defines severe ARDS for a patient on positive end-expiratory pressure (PEEP) $\geq 5 \mathrm{cmH}_{2} \mathrm{O}$ [16]. For patients diagnosed with COVID-19, ARDS has been evident in $3.4 \%$ of hospitalized patients and $60-70 \%$ of intensive care unit (ICU) patients $[17,18]$. A recent analysis of typical ARDS compared to COVID-19-related ARDS revealed differences in onset timing, respiratory system compliance, disease severity based on oxygenation indices, and appropriate management protocols [2].

Following patient stacking methods recommended by Neyman et al. and Paladino et al., there are many concerns with MPV [4, 6]. Several medical associations published a joint article advising medical personnel against such practices [19]. Their reasons for issuing such a statement included: tidal volume titration is not possible as the largest volume will go to the most compliant lungs, individual patient pulmonary mechanics monitoring is difficult and may require additional external monitors, removing or adding patients to the combined circuit poses a risk for all other patients and ethical issues where testing clinically unproven MPV risks life-threatening treatment failure. To implement MPV in patients with ARDS, a mechanism to address these issues is necessary.

The objective of this study was to address ventilator shortages and patient stacking limitations by developing and validating a modified breathing circuit for two patients with differing lung compliances using simple, off-the-shelf components. The proposed solution combined two individual patient circuits and added a precision flow resistor (FRE) to both inspiratory limbs to create a multi-patient ventilator circuit (MPVC). The MPVC was simulated with a mathematical model (Supplemental Material) and validated with four studies using two human-sized pigs each: one healthy and one with lipopolysaccharide (LPS) induced ARDS. To our knowledge, this is the first study that has validated an MPVC using an ARDS animal injury model. The results of this study found that two pigs with different lung compliances could be adequately oxygenated on a single ventilator for 4 hours with minor changes to patient circuits.

\section{Methods}

To enable patient stacking during pressure control mode, two individual patient circuits were combined, and a flow resistor was placed on each inspiratory limb. The result of these changes was a multi-patient ventilator circuit (MPVC) where the FRE could be adjusted to restrict flow to the healthier patient $(\mathrm{PH})$ to prevent over-ventilation. Each patient had an FRE on their inspiratory limb to match nominal circuit resistances and for instances when PS improved faster than $\mathrm{PH}$.

\section{Circuit setup}

Since the mathematical model showed the feasibility of accommodating two patients on a single ventilator, a novel ventilator setup was designed based on the model (Fig. 1). The circuit setup for two patients on an open-circuit ventilator (such as the Newport HT70 Plus ventilator used in this research) is shown in Fig. 1A. Each patient is connected to a standard full circuit which includes an inhalation limb, patient wye connector, endotracheal tube, exhalation valve/ tubing, and a proximal line. The extra parts needed for multipatient ventilation are outlined in Table 1.

As shown in Fig. 1, the inhalation limb was separated with a tee connector. Each split inhalation line ran to an FRE. Both patient circuits included an FRE so the nominal resistance through each circuit was equal and in the event the patients' lung compliance differences were reversed. Before the FRE was inserted, an adapter (not shown in setup) was used as a place holder. To change which patient is being monitored with the proximal line, a three-way flow valve connected the lines to the ventilator. Both exhalation valve control lines were joined with a wye connector to simultaneously actuate each valve. An MPV simulation was done using two adjustable precision test lungs with modular lung compliance and airway resistance (QuickLung, IngMar Medical, Pittsburg, PA). The benchtop test showed the functionality of ventilating two patients with differing injury severities using the MPVC. 

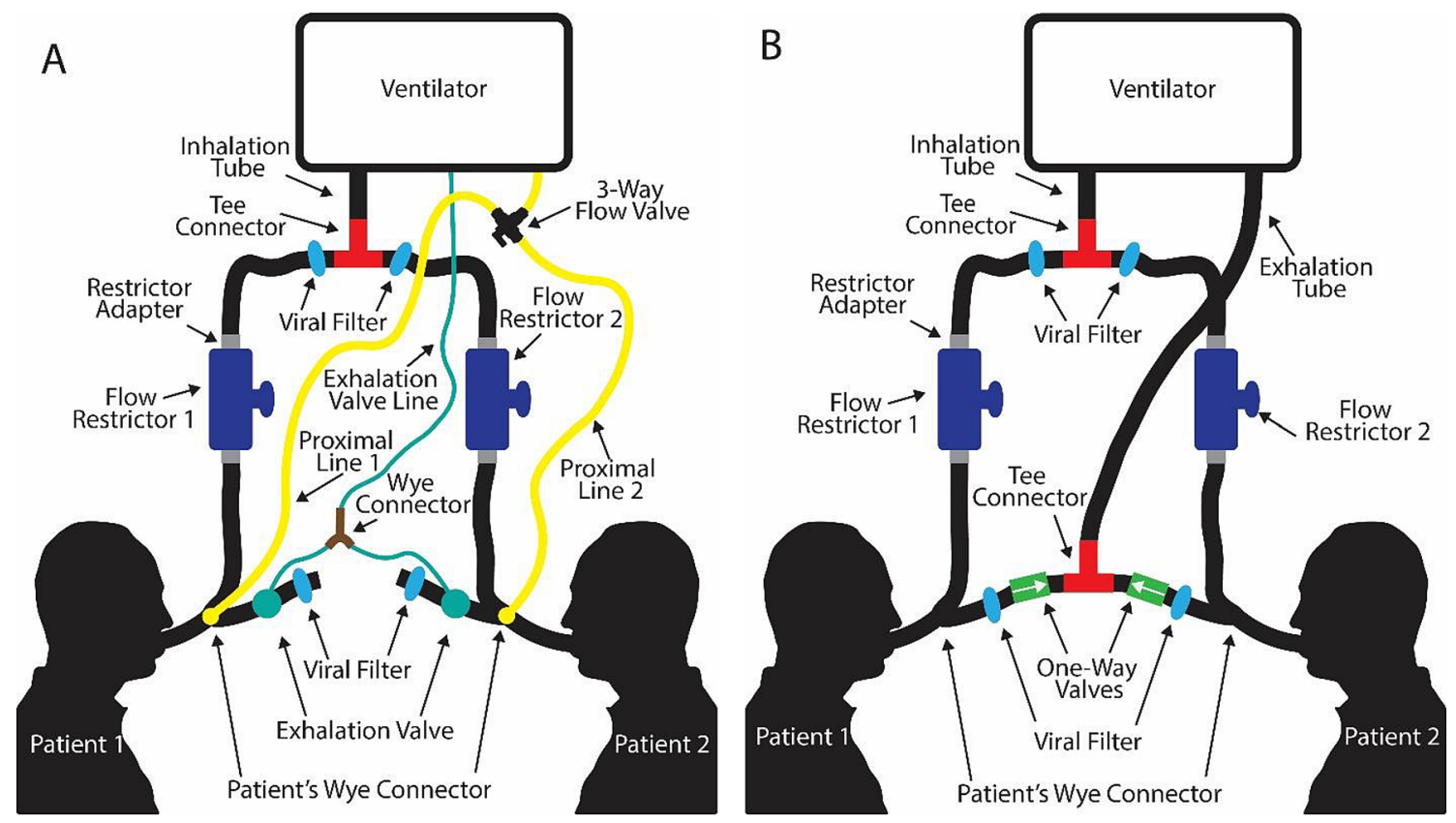

Fig. 1 Schematic setup for the MPVC on A an open-loop ventilator and $\mathbf{B}$ a closed-loop ventilator

Table 1 Extra components needed for dual-patient ventilation

\begin{tabular}{ll}
\hline Part & Function \\
\hline Tee connector & Split the breathing circuit \\
Flow restrictor $\times 2$ & Titrate flow \\
Wye connector & Joins the two exhalation valve lines back to the ventilator \\
3-way flow valve & Allows specified patient to be monitored via the proximal line \\
Flow restrictor to tube adapter & Enable use of the flow restrictors \\
Circuit adapters (not in final setup) & Acts as a place holder to connect the two ends of the breath- \\
& ing circuit where the FRE will be placed \\
Respiratory profile monitor & Monitor both patients \\
Viral filter $\times 4$ & Prevent viral spreading between patients \\
\hline
\end{tabular}

Although the studies were performed with an open-loop ventilator (Fig. 1A), the same concept can be applied to a closed-loop ventilator Fig. 1B Since the exhalation line flows back to the ventilator in closed-loop set-ups, the exhalation tubes are joined with the same tee connector used for joining the inhalation lines. Each patient also has a one-way valve on their exhalation limb to prevent the backflow of air.

\section{Animal study}

All animal studies were performed in accordance with the University of Nebraska-Lincoln Institutional Animal Care and Use Committee (IACUC), NIH Guide for the Care and Use of Laboratory Animals, ARRIVE guidelines, and the Animal Care and Use Review Office (ID \# 1944). Humansized female Duroc cross pigs were used for their comparable size and anatomy. All animals were received for acclimation 4 days before the experiment, group-housed in open pens, and given a toy for enrichment. Eight pigs were divided into healthier patient $(\mathrm{PH})(n=4$, mass $=73 \pm 3 \mathrm{~kg})$ and sicker (ARDS) patient (PS) $(n=4$, mass $=75 \pm 3 \mathrm{~kg})$ groups. An additional subset of female Duroc cross pigs ( $n=4$, mass $=45 \pm 1 \mathrm{~kg}$ ) was used as single patient ventilation circuit (SPVC) animals. No randomization was done between the SPVC and the MPVC animals. However, the PS animal was randomly selected for the MPVC studies. All surgeries were performed at the Life Sciences Annex at the University of Nebraska-Lincoln.

The experiment was performed in three stages. In the first stage, two pigs underwent the surgical placement of catheters at staggered time intervals. In the second stage, one pig was given nebulized LPS to induce ARDS while the other pig was maintained. In the third stage, the ARDS pig was moved to the healthier pig's ventilator and both pigs were 
ventilated together for 4 hours. A total of four experiments were performed. The first two experiments aimed to develop the protocol for multi-patient ventilation. The second two experiments aimed to maintain end-tidal $\mathrm{CO}_{2}$ between 30 and $40 \mathrm{mmHg}$ for both pigs by adjusting the PH's FRE and the ventilator settings. More information about the surgery and ARDS protocols can be found in the supplemental materials.

\section{Statistics and data analysis}

Statistical comparisons of $\mathrm{PaO}_{2}$ and $\mathrm{SpO}_{2}$ between the SPVC and MPVC animals were performed in Microsoft Excel 365. A power analysis with an estimated difference in mean $\mathrm{PaO}_{2}$ levels between the MPVC and SPVC animals of $20 \mathrm{mmHg}$ and a power of 0.8 was used to determine four studies were necessary. A two-sample heteroscedastic $t$ test was performed on oxygen levels at 30-min intervals with a significance level of $\alpha=0.05$.

\section{Results}

\section{In-vivo study with the multi-patient ventilation circuit}

Four trials were performed where a pig that developed moderate ARDS (PS) from LPS was attached to the MPVC with a healthy animal $(\mathrm{PH})$, thus maximizing a difference in lung compliance. The animal injury model was developed using LPS, following the procedure described in a previous study by Lutz et al. [20]. However, the LPS dose of $4 \mathrm{mg} / \mathrm{kg}$ used in the present study was slightly higher than the dose previously used by other groups [21-25]. The PS in studies one and three survived to the experiment endpoint, and the PS in trials two and four experienced cardiac arrests before the four-hour experiment endpoint. During all four experiments, the PHs maintained stable vital signs and showed no signs of trauma from over-ventilation. A controlled study was performed with four PSs placed on single patient ventilator circuits (SPVC). Of the four SPVC animals, one never developed moderate to severe ARDS after receiving $4 \mathrm{mg}$ / $\mathrm{kg}$ of LPS and was thus excluded from comparisons. Of the remaining three animals, two survived until the end of the experiment.

\section{Individual ventilation vs. multi-patient ventilation blood oxygenation}

The average oxygenation levels in partial pressure of oxygen $\left(\mathrm{PaO}_{2}\right)$ and peripheral oxygen saturation $\left(\mathrm{SpO}_{2}\right)$ of the PS animals on MPVC were compared to the SPVC animals. This was done by aligning the experiment time based on the time each animal met the Berlin criteria for moderate to severe ARDS. Because some animals died earlier than the 4-h endpoint and others took longer to develop ARDS, the number of animals (n values located just above the $x$-axis in Fig. 2) compared at each 30-min time point varied. Three baseline measurements taken before LPS administration were compared. The $\mathrm{SpO}_{2}$ values for pigs on SPVC had a range of $68-89 \%$ during the 4-hour study. Similarly, the $\mathrm{SpO}_{2}$ values for pigs on MVPC had a range of 70-93\%. The $\mathrm{SpO}_{2} / \mathrm{FiO}_{2}$ ratio for pigs on SPVC and MPVC ranged from 74 to 94 and 50 to 108 , respectively. No statistically significant differences were found in $\mathrm{PaO}_{2}$ throughout the study ( $p>0.05$ ) (Fig. 2A). For $\mathrm{SpO}_{2}$ (Fig. 2B), $t+0.5$ h. was the only significant difference $(p<0.001)$. As seen in Fig. 2, the $\mathrm{SpO}_{2}$ level drops immediately after ARDS development which is also the time the stacked ventilator pigs were switched to the MPVC.

\section{Results from the multi-patient in-vivo experiments}

Vital signs, ventilator changes, and FRE adjustments for PH and PS were recorded for the duration of each trial. Figure 3 illustrates the timeline of events and vital signs recorded. Peripheral oxygen saturation $\left(\mathrm{SpO}_{2} ; \%\right)$, heart rate (bpm), mean arterial pressure (MAP; $\mathrm{mmHg}$ ), end-tidal carbon dioxide $\left(\mathrm{EtCO}_{2} ; \mathrm{mmHg}\right)$, positive end-expiratory pressure (PEEP, $\mathrm{cmH}_{2} \mathrm{O}$ ), partial pressure of oxygen to fraction of inspired oxygen (Berlin Criteria), respiratory rate (breaths/ $\mathrm{min})$, minute volume $(\mathrm{L} / \mathrm{min})$, peak pressure $\left(\mathrm{cmH}_{2} \mathrm{O}\right)$, tidal volume $(\mathrm{mL})$, and FRE resistance were measured. $\mathrm{EtCO}_{2}$ data were not collected for PS during LPS administration. The ventilator pressure control, PEEP, respiratory rate, minute volume, peak pressure, and tidal volume were recorded for each animal separately until they were placed on the MPVC. After MPV began, reported minute and tidal volumes were the sum of the two patients' volumes. In studies 1 and 3, both PH and PS survived to the 4-h endpoint of the trial. In studies 2 and 4, PS died before the 4-hour endpoint, and $\mathrm{PH}$ was subsequently euthanized.

During the MPVC trials, it was noted that when PS was unstable, $\mathrm{PH}$ remained stable. After the start of MPV in study $1, \mathrm{PH}_{1}$ (where the subscript denotes the study number 1-4) had adequate oxygenation with $\mathrm{PaO}_{2}$ levels that remained above $225 \mathrm{mmHg}$ and $\mathrm{SpO}_{2}$ levels above $95 \%$. $\mathrm{PS}_{1}$ 's $\mathrm{PaO}_{2}$ values remained below $100 \mathrm{mmHg}$ for approximately $3.5 \mathrm{~h}$. Rotating the animals from supine to lateral position appeared to decrease their $\mathrm{EtCO}_{2}$ levels. In study 3 , the first baseline $\mathrm{PaO}_{2}$ measurements were low because the oxygen tank was mistakenly left unopened. The three baseline measurements were subsequently repeated.

After the start of MPV in study 4, $\mathrm{PS}_{4}$ exhibited a fluctuating bpm and $\mathrm{EtCO}_{2}$ before circuit combination. Instead of starting MPV immediately after the Berlin criteria was 

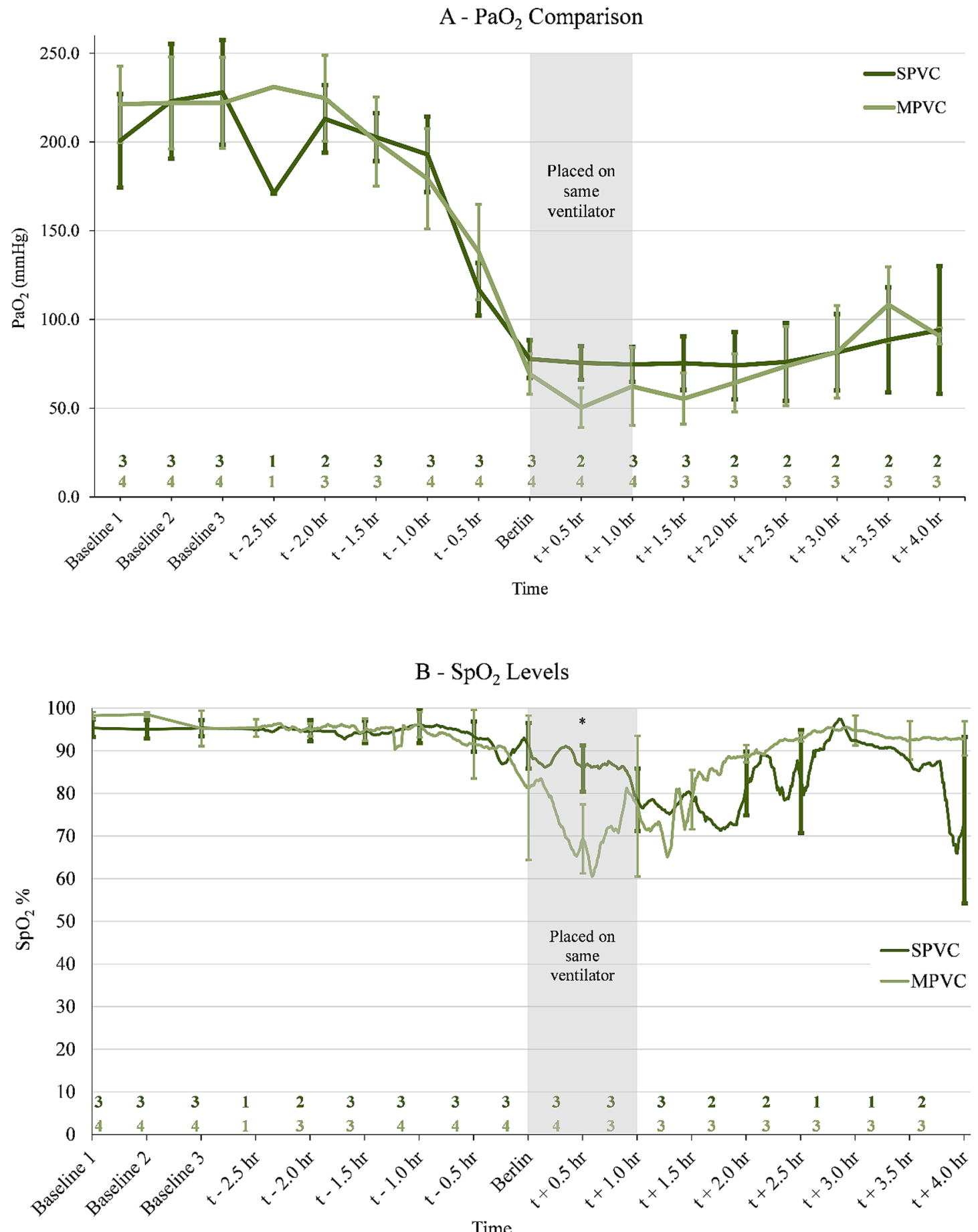

Fig. 2 There is no statistically significant difference in $\mathrm{PaO}_{2}$ values (A) and one difference in $\mathrm{SpO}_{2}$ values $(p<0.001$, indicated by *) (B) when comparing individually ventilated pigs (SPVC) with patient stacked pigs (MPVC). Oxygen comparison between three ARDS animals that were individually ventilated and four ARDS animals that were ventilated along with a healthy animal during ARDS development and up to 4 hours after is shown. A comparison between the two groups was performed with a two-sample heteroscedastic $t$ test with a significance level of $\alpha=0.05$. The sample size ( $n$ ) for each time step is given above the $x$-axis. Since some animals developed ARDS faster than others and some died before reaching the end of the study, $n$ values (located just above the $x$-axis) varied but were centered around developing moderate to severe ARDS per the Berlin criteria. Three baseline measurements were taken before any LPS administration and were compared together at the beginning of the chart. The stacked ventilation pigs were placed on the same ventilator within 1 hour of reaching ARDS. Error bars indicate $\pm 1 \mathrm{SD}$ 


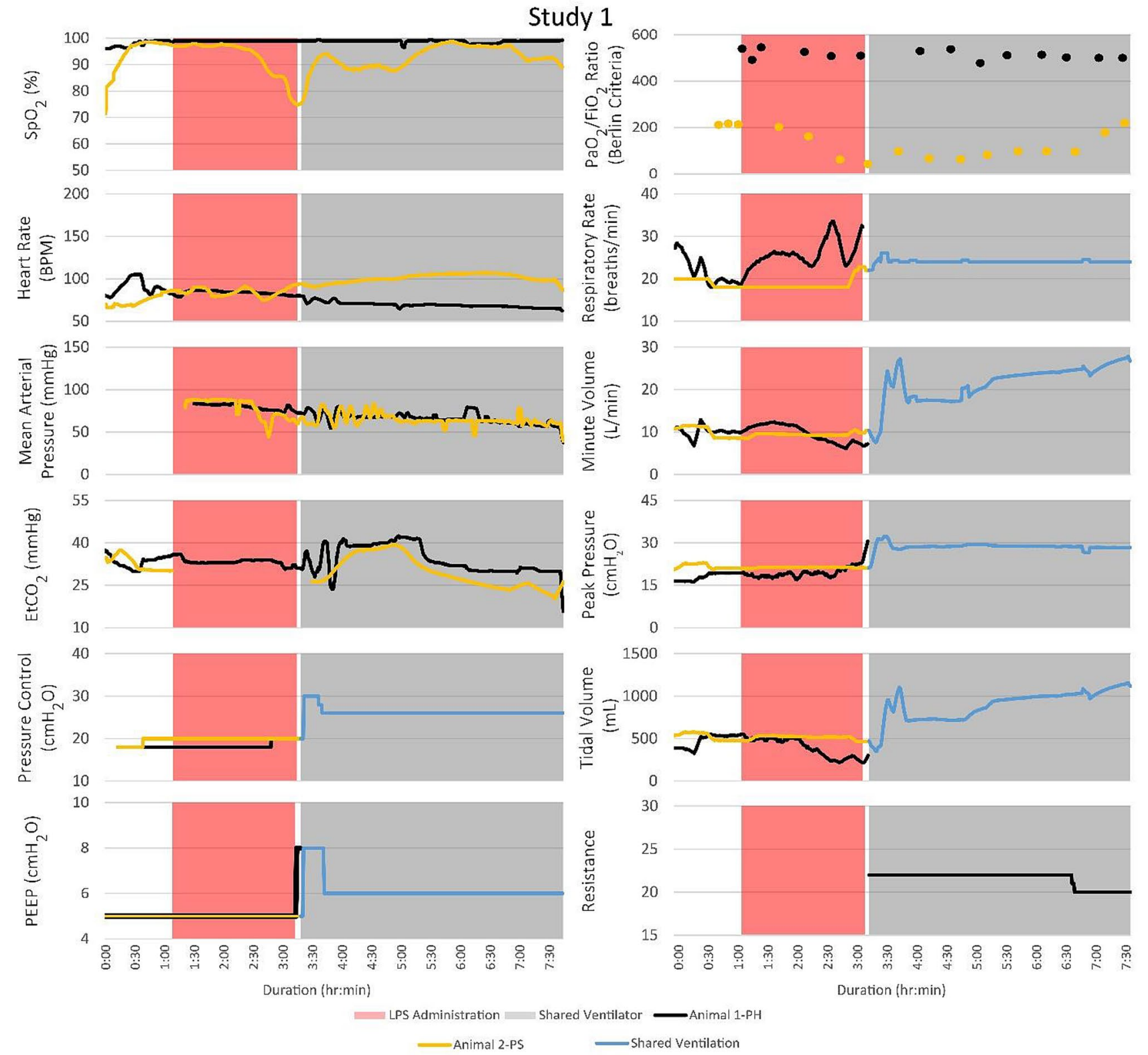

Fig. 3 Although the PSs became unstable in every study and required ventilator adjustments, the PHs that were attached to the same ventilator remained stable. The timelines for vital signs and events for each of the four studies are shown. Peak pressure adjustments of the venti-

met, 40 minutes was allowed to stabilize $\mathrm{PS}_{4}$ before being placed on the MPVC. $\mathrm{PH}_{4}$ exhibited $\mathrm{PaO}_{2}$ levels above $225 \mathrm{mmHg}, \mathrm{SpO}_{2}$ levels above $90 \%$, stable bpm and MAP during MPV. $\mathrm{PS}_{4}$ was extremely unstable with fluctuating bpm, decreasing oxygenation and MAP before going into cardiac arrest after $3 \mathrm{~h}$ of ventilation on the MPVC. Many ventilator pressure changes were made to recover the animal, but little response was seen. The $\mathrm{SpO}_{2}$ levels fell as low as $47 \%$. After $40 \mathrm{~min}$ of treatment via atropine injection, repositioning from supine to lateral, and increasing PEEP, lator significantly impacted the PSs with minimal impact on the PHs. FRE adjustments affected the vital signs of the PHs with no noticeable effect on the PSs

$\mathrm{PH}_{4}$ did not stabilize and was placed on the MPVC. For the remainder of study 4 , the ventilator pressure was adjusted to a maximum of $30 \mathrm{mmHg}$ to adequately oxygenate $\mathrm{PS}_{4}$ while $\mathrm{PH}_{4}$ remained stable with the FRE restricting airflow.

In general, decreasing the FRE's resistance resulted in decreased $\mathrm{EtCO}_{2}$ for $\mathrm{PH}$ and vice versa when increasing the FRE's resistance. The $\mathrm{EtCO}_{2}$ level of $\mathrm{PH}_{1}$ was unstable after being put on the MPVC and was above $40 \mathrm{mmHg}$ after leveling out. Table 2 lists the observed changes in $\mathrm{EtCO}_{2}$ levels after changes were made to ventilator settings or the FRE. For 


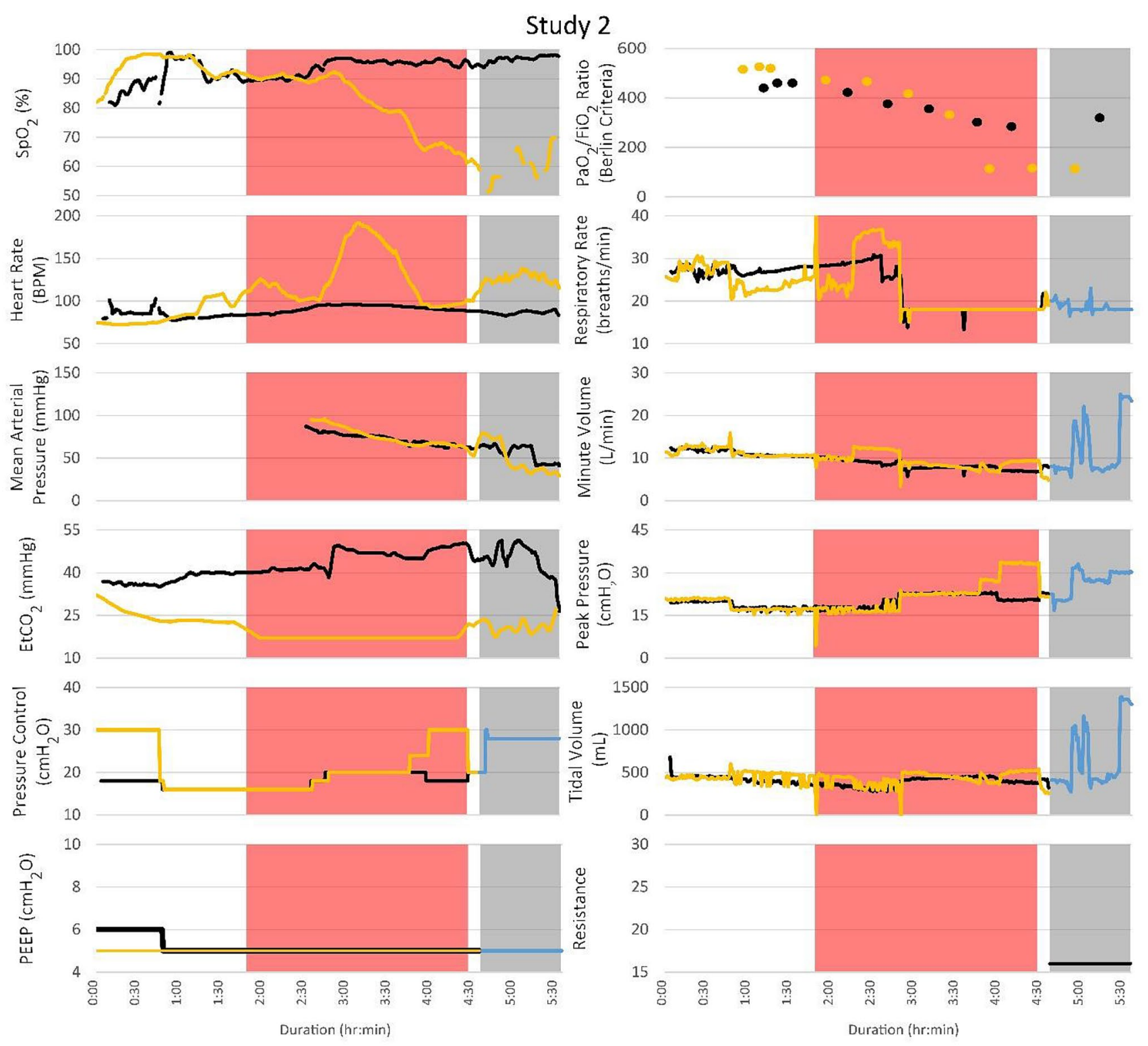

LPS Administration Shared Ventilator $\longrightarrow$ Animal 3-PH

Fig. 3 (continued)

Table 2, PS's proximal line was monitored by the ventilator. Each patient has an FRE; however, changing the patient's FRE that's proximal line is being monitored (PS in this case) does not affect since the ventilator is set to match that patient's pressure control (PC) setting. Thus, the FRE on the PH's circuit is the only FRE adjusted unless the patient's lung compliances are switched.

\section{Discussion}

The objective of this study was to address ventilator shortages and patient stacking limitations by developing and validating a modified breathing circuit for two patients with different lung compliances. An important component 


\section{Study 3}

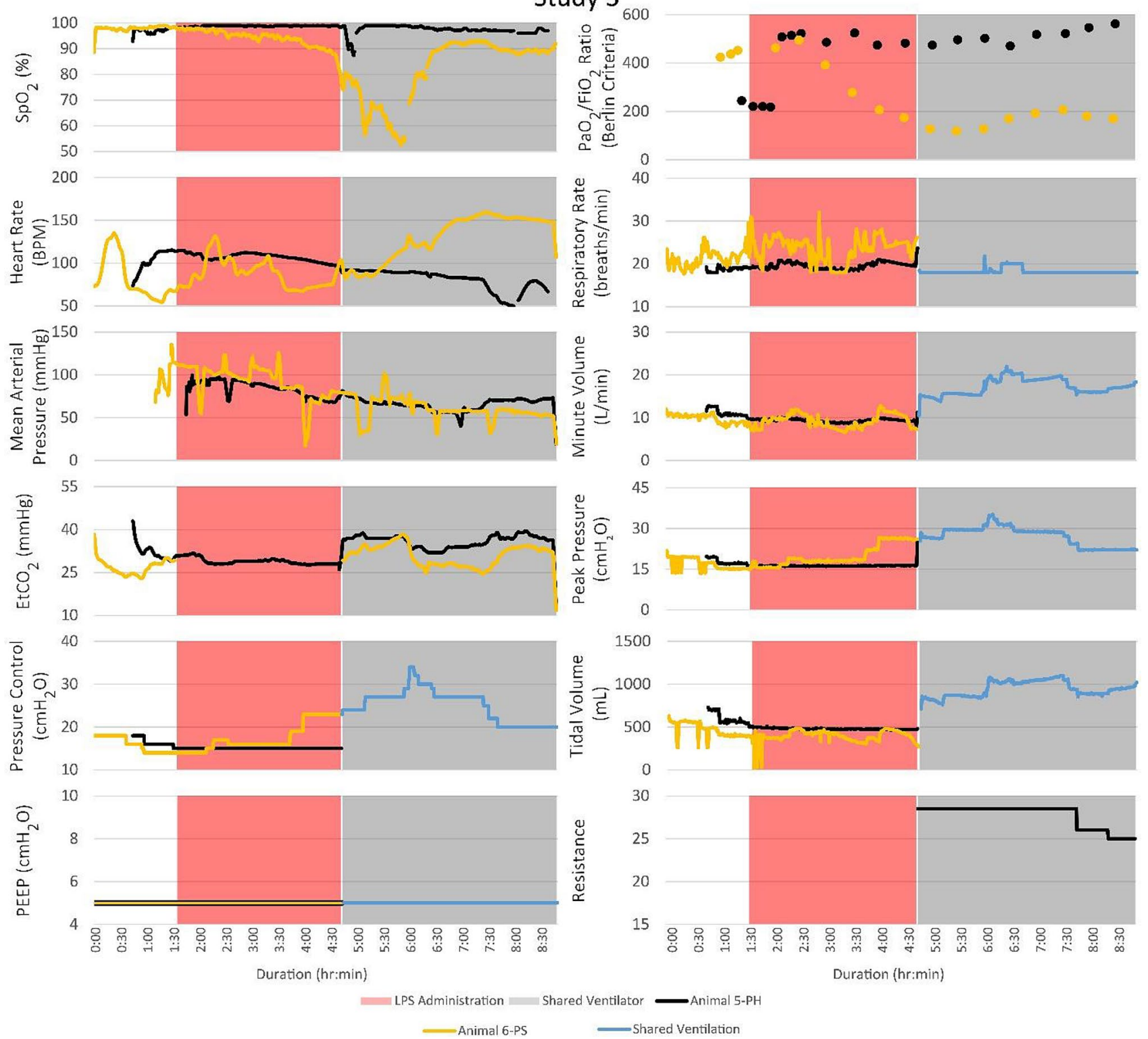

Fig. 3 (continued)

of this study was to test the technique on an animal injury model of ARDS. The efficacy of the MPVC design was tested using a porcine model of LPS-induced ARDS as a model for COVID-19. There are several characteristics of COVID-19 induced ARDS that are approximated by the LPS-induced ARDS model. Most notably poor oxygenation, which COVID-19 patients may encounter despite relatively normal lung compliance [26]. As the disease progresses, inflammation and edema in the lungs increases, leading to a decrease in lung compliance. The porcine model used in this study consistently presented hypoxemia. As such, the range of $\mathrm{PaO}_{2} / \mathrm{FiO}_{2}$ for both the SPVC and MPVC ARDS animals was consistent with hypoxemia that characterizes moderate to severe ARDS. This model also presented inflammation in the lung tissue which contributes to edema in infected lungs [26, 26]. The LPS-induced ARDS model had a rapid onset of $<2 \mathrm{~h}$ and was representative of an advanced stage of ARDS.

This study found that there were no significant differences in oxygenation between SPVC and MPVC animals. As the animals were placed on the same ventilator, they were without oxygen support for a short period. This is a possible explanation for the difference in $\mathrm{SpO}_{2}$ values at $t+0.5 \mathrm{~h}$. between the MPVC and SPVC ARDS animals. An alternative explanation for the difference in oxygenation is the $100 \% \mathrm{FiO}_{2}$ administered to only the SPVC animals during 


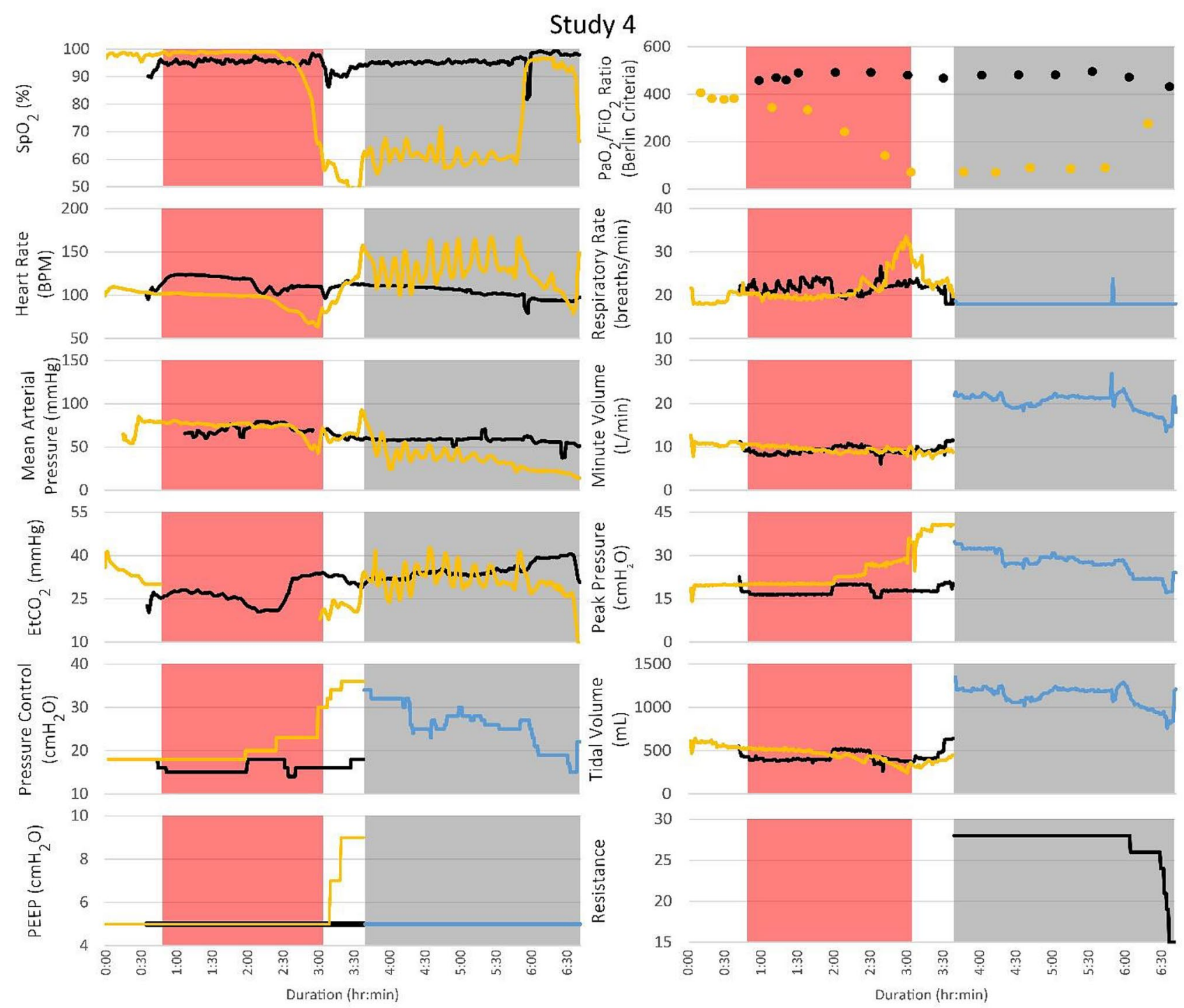

LPS Administration $—$ Shared Ventilator —Animal 7-PH

Fig. 3 (continued)

Table 2 The impact of ventilator and FRE changes on the MPVC setup

\begin{tabular}{|c|c|c|}
\hline Action & PS effect & PH effect \\
\hline Increase ventilator pressure control (PC) & Decrease $\mathrm{EtCO}_{2}$ & Decrease $\mathrm{EtCO}_{2}$ \\
\hline Decrease ventilator PC & Increase $\mathrm{EtCO}_{2}$ & Increase $\mathrm{EtCO}_{2}$ \\
\hline $\begin{array}{l}\text { Increase ventilator positive end expiratory } \\
\text { pressure (PEEP) }\end{array}$ & Minimal increase $\mathrm{EtCO}_{2}$ & Minimal increase $\mathrm{ETCO}_{2}$ \\
\hline Decrease ventilator PEEP & Minimal decrease $\mathrm{EtCO}_{2}$ & Minimal decrease $\mathrm{EtCO}_{2}$ \\
\hline Decrease PH’s FRE resistance & No effect & Decrease $\mathrm{EtCO}_{2}$ \\
\hline Increase PHs FRE resistance & No effect & Increase $\mathrm{EtCO}_{2}$ \\
\hline Increase ventilator I-time & Decrease $\mathrm{EtCO}_{2}$ & Decrease $\mathrm{EtCO}_{2}$ \\
\hline Decrease ventilator I-time & Increase $\mathrm{EtCO}_{2}$ & Increase $\mathrm{EtCO}_{2}$ \\
\hline
\end{tabular}

For the standard MPVC setup, PS has the proximal line attached to give feedback to the ventilator (I.e., the three-way flow valve is turned to monitor PS). Each patient has their own FRE; however, adjusting the PS's FRE has no effect since the proximal line is monitoring that patient. Thus, the FRE on the PH circuit is the only FRE that should be adjusted unless the patient's lung compliances are switched 
the bronchoscopies. On average, bronchoscopy duration was 6 min and occurred 15 min after the Berlin criteria for moderate ARDS Berlin was reached. Other than the single $t+0.5 \mathrm{~h}$. time point, oxygenation levels between the two groups were similar. Additionally, this study demonstrated an extreme lung compliance variation as $\mathrm{PH}$ was ventilated alongside PS. Thus, this model showed that the MPVC can be used to ventilate two patients with mismatched lung compliances and provide sufficient oxygenation.

Due to the difference in lung compliance between $\mathrm{PH}$ and PS, PH does not require the same airflow and pressure that PS does. Since the $\mathrm{FiO}_{2}$ is the same for both patients, a balance must be found between keeping PS oxygenated but not over-ventilating PH. When the resistance of the healthier animal's FRE was increased, it restricted airflow and thus prevented the patient from being over-ventilated which could cause overstretching of the lungs, hyperoxia, and inflammation. Adjusting the FRE had no noticeable effect on PS. Changes in ventilator pressure control were made to ensure adequate gas exchange in PS, which was evaluated by $\mathrm{PaO}_{2}$, $\mathrm{SpO}_{2}$, and $\mathrm{EtCO}_{2}$. $\mathrm{PH}$ remained stable despite these changes in ventilator pressure. The tolerance of $\mathrm{PH}$ to the MPVC was demonstrated in study 4 where PS became extremely unstable and frequent changes in ventilator pressure were made. The FRE showed to be effective in preventing over-ventilation of the PH throughout all ventilator changes made in an attempt to stabilize PS. $\mathrm{PH}$ 's $\mathrm{PaO}_{2}$ values were elevated to $250 \mathrm{mmHg}$, which can be acceptable for a moderate period.

There are some limitations to MPV. A known method of ventilating ARDS patients is airway pressure release ventilation. This method uses high continuous airway pressure to promote alveolar recruitment [26]. However, this method is not possible when ventilating PH since FRE slows inhalation. As a result, $\mathrm{PH}$ never reaches a sustained peak pressure (Fig. 2E). This problem may be overcome by increasing PEEP to raise the mean airway pressure and enable more oxygenation. Also, certain ventilator parameters, such as respiratory rate, I-time, PEEP, and $\mathrm{FiO}_{2} \%$ cannot be individualized for each patient using our MPVC. However, using an FRE one can individualize PC and tidal volume. Srinivasan et al. were able to overcome part of this problem by individualizing PEEP using PEEP valves on each patient's expiratory line [5]. Additionally, our MPVC setup did not include any tidal volume measurements. Changes to the ventilator and FRE were based solely on vital readings. However, for ARDS patients, maintaining low tidal volumes is one of the only proven methods for successful treatment [26]. To obtain a tidal volume measurement, an external respiratory monitor would be required.

Another limitation of the presented study was respiratory system compliance of PS and PH were not explicitly measured. However, the low lung compliance of the ARDS animals was evident in the high peak pressure required to sustain life after receiving LPS. As seen in every study in Fig. 3, before receiving LPS, both animals were stable at a pressure control setting between 15 and $20 \mathrm{cmH}_{2} \mathrm{O}$. After LPS administration, pressure control settings were mainly between 25 and $30 \mathrm{cmH}_{2} \mathrm{O}$. These higher pressures were required to sufficiently oxygenate the ARDS animal. Also, this difference in the pressure setting requirement between the two animals (nothing was given to the healthy animal so its pressure setting requirement should have remained stable) highlights the need for a flow or pressure titration device.

The animal study showed it is possible to ventilate multiple subjects on a single ventilator for a short period using simple flow restrictors and minor tubing set modifications. The presented method should only be considered during ventilator shortages. Additionally, to practically implement MPV in a medical center, the ventilator alarms need to be set up to alert health care personnel of ventilation issues. Srinivasan et al. addressed this by setting the exhaled volume and minute ventilation alarms according to the sum of the patients' tidal volumes [5]. They enabled the ventilator to alarm in response to any circuit occlusions, shunts, or disconnections from endotracheal tubes [5]. Srinivasan et al. also experimentally showed that the extra viral filters attached to each patient's inspiratory limbs prevents crosscontamination (Fig. 1) [5]. They showed this by nebulizing trypan blue into the inspiratory limb of an artificial lung and monitoring the flow paths. They concluded that no crosscontamination was visually observed or detected from wipe tests of each segment of the circuit.

For the MPVC setup, the precision needle valve sufficiently regulated the pressure to the healthy patient. A precision resistor with high resolution and low nominal resistance is necessary to fine-tune ventilation pressure and to avoid circuit occlusion alarms. Clarke et al. used a Hoffman clamp on a tracheal tube connected in series with an inspiratory limb of their test lungs [7]. Hoffman clamps are readily available in most hospitals. However, unlike a precision needle valve, they do not allow a high titration resolution.

An FRE on each patient's inspiratory limb is necessary as patients may progress or digress at different rates. In such a case, the healthier patient may become the sicker patient. Because of this, the now healthier patient's FRE should be adjusted to compensate for over-ventilation, and the now sicker patient's proximal line should be monitored and its FRE opened completely. While ventilating multiple patients, it may be necessary to place one or both patients in the prone position or to remove a patient from the MPVC (e.g., to perform a spontaneous breathing test). Although the animals in this study were not placed in the prone position, they were rotated between the left and right lateral recumbent positions. Thus, placing a patient in the prone position should also be possible. Switching a patient to single ventilation is as simple as detaching the patient from the MPVC at the tee and wye connectors 
and adding caps in their place, replacing the FRE with the circuit tube adapter, and ensuring the three-way valve is monitoring the single patient. Once the patients have been separated, a spontaneous breathing test can be performed as usual [26]. For this reason, there must be extra ventilators available to practically implement MPV within a hospital or clinic".

Although the study was only performed on two porcine subjects, the principles could likely be applied to ventilating more than two patients. Theoretically, the volume capacity of a ventilator is the main limiting factor to the number of patients that could be placed onto a single ventilator. The average tidal volume capacity for a hospital ICU ventilator is around $2500 \mathrm{~mL}$. Assuming the average person will need a tidal volume of $500 \mathrm{~mL}$ [26], up to 5 patients could be on a single ventilator.

We have demonstrated how multiple patients with differing respiratory impedances can be ventilated with a single ventilator and receive sufficient respiratory support without overventilating PH. As healthcare systems worldwide deal with inundated ICUs and hospitals from the COVID-19 pandemic, they could potentially benefit from this type of system by enabling respiratory care to more patients with ARDS.

Supplementary Information The online version contains supplementary material available at https://doi.org/10.1007/s00540-021-02948-2.

Acknowledgements The animal handling and surgical work were supported by the Institutional Animal Care staff, veterinarians, and technicians from the University of Nebraska-Lincoln.

Author contributions BW, RR, AM: data acquisition, data analysis, data interpretation, manuscript drafting, manuscript revising. NZ, KB: data acquisition, data analysis, data interpretation, manuscript revising. RS, FF, TF: data interpretation, manuscript revising. BT: conception of the work, data analysis, data interpretation, manuscript revising.

Funding This study was funded by the University of Nebraska-Lincoln College of Engineering and the University of Nebraska Office of Research and Economic Development. Funds were awarded to BST. The funders had no role in study design, data collection and analysis, decision to publish, or preparation of the manuscript. UNL College of Engineering: https://engineering.unl.edu/. UNL Office of Research and Economic Development: https://research.unl.edu/.

\section{Declarations}

Conflict of interest Ben Wankum, Riley Reynolds, Andrea McCain, Nathaniel Zollinger, Keely Buesing, Russel Sindelar, Frank Freihaut, Tariku Fekadu, and Ben Terry have no conflicts of interest or financial ties to disclose.

\section{References}

1. Dreher M, Kersten A, Bickenbach J, Balfanz P, Hartmann B, Cornelissen C, Daher A, Stöhr R, Kleines M, W. Lemmen S, Christian Brokmann J, Müller T, Müller-Wieland D, Marx G, Marx N. The characteristics of 50 hospitalized
COVID-19 patients with and without ARDS. Dtsch Arztebl Int. 2020;117:271-8.

2. Li X, Ma X. Acute respiratory failure in COVID-19: is it "typical" ARDS? Crit Care. 2020;24:198.

3. Fried MW, Crawford JM, Mospan AR, Watkins SE, Munoz B, Zink RC, et al. Patient characteristics and outcomes of 11721 patients with Coronavirus Disease 2019 (COVID-19) hospitalized across the United States. Clin Infect Dis. https://academic. oup.com/cid/advance-article/doi/10.1093/cid/ciaa1268/58982 76. Accessed 4 Dec 2020

4. Neyman G, Irvin CB. A single ventilator for multiple simulated patients to meet disaster surge. Acad Emerg Med. 2006;13:1246-9.

5. Srinivasan S, Ramadi KB, Vicario F, Gwynne D, Hayward A, Lagier D, et al. A rapidly deployable individualized system for augmenting ventilator capacity. Science Translational Medicine. American Association for the Advancement of Science. 2020. https://stm.sciencemag.org/content/early/2020/05/18/scitranslm ed.abb9401. Accessed 1 Jun 2020

6. Paladino L, Silverberg M, Charchaflieh JG, Eason JK, Wright BJ, Palamidessi N, Arquilla B, Sinert R, Manoach S. Increasing ventilator surge capacity in disasters: ventilation of four adult-human-sized sheep on a single ventilator with a modified circuit. Resuscitation. 2008;77:121-6.

7. Clarke AL, Stephens AF, Liao S, Byrne TJ, Gregory SD. Coping with COVID-19: ventilator splitting with differential driving pressures using standard hospital equipment. Anaesthesia. 2020;75:872-80.

8. Smith R, Brown JM. Simultaneous ventilation of two healthy subjects with a single ventilator. Resuscitation. 2009;80:1087.

9. How One Las Vegas ED saved hundreds of lives after the worst mass shooting in US History. https://epmonthly.com/article/ not-heroes-wear-capes-one-las-vegas-ed-saved-hundreds-livesworst-mass-shooting-u-s-history/. Accessed 19 Jun 2020

10. Beitler JR, Mittel AM, Kallet R, Kacmarek R, Hess D, Branson $\mathrm{R}$, et al. Ventilator sharing during an acute shortage caused by the COVID-19 pandemic. Am J Respir Crit Care Med. 2020;202:600-4.

11. Pontoppidan H, Geffin B, Lowenstein E. Acute respiratory failure in the adult. N Engl J Med. 1972;287:690-8.

12. Chiumello D, Froio S, Mistraletti G, Formenti P, Bolgiaghi L, Cammaroto A, Umbrello M, Coppola S. Gas exchange, specific lung elastance and mechanical power in the early and persistent ARDS. J Crit Care. 2020;55:42-7.

13. Wright PE, Bernard GR. The role of airflow resistance in patients with the adult respiratory distress syndrome. American Review of Respiratory Disease. American Lung Association. 2012. https://www.atsjournals.org/doi/pdf/10.1164/ajrccm/ 139.5.1169. Accessed 28 May 2020

14. Sklar MC, Fan E, Goligher EC. High-frequency oscillatory ventilation in adults with ARDS: past, present, and future. Chest. 2017;152:1306-17.

15. Peck TJ, Hibbert KA. Recent advances in the understanding and management of ARDS. F1000Research. 2019;8:1959.

16. Syndrome ARD. The Berlin definition. JAMA. 2012;307:2526-33.

17. Guan W, Ni Z, Hu Y, Liang W, Ou C, He J, et al. Clinical characteristics of coronavirus disease 2019 in China. New England Journal of Medicine. Massachusetts Medical Society. 2020. https:// www.nejm.org/doi/10.1056/NEJMoa2002032. Accessed 3 Jun 2020

18. Phua J, Weng L, Ling L, Egi M, Lim C-M, Divatia JV, Shrestha BR, Arabi YM, Ng J, Gomersall CD, Nishimura M,Koh Y, $\mathrm{Du}$ B. Intensive care management of coronavirus disease 2019 (COVID-19): challenges and recommendations. Lancet Respir Med. 2020;8:506-17. 
19. Joint statement on multiple patients per ventilator [Internet]. https://www.asahq.org/about-asa/newsroom/news-releases/2020/ 03/joint-statement-on-multiple-patients-per-ventilator. Accessed 15 Apr 2021

20. Lutz C, Carney D, Finck C, Picone A, Gatto LA, Paskanik A, et al. Aerosolized surfactant improves pulmonary function in endotoxin-induced lung injury. American Journal of Respiratory and Critical Care Medicine. 1998;158:840-5.

21. Hochhausen N, Orschulik J, Follmann A, Santos SA, Dohmeier H, Leonhardt $\mathrm{S}$, et al. Comparison of two experimental ARDS models in pigs using electrical impedance tomography. PLoS ONE. 2019;14:1-14.

22. Fu H, Sun M, Miao C. Effects of different concentrations of isoflurane pretreatment on respiratory mechanics, oxygenation and hemodynamics in LPS-induced acute respiratory distress syndrome model of juvenile piglets. Experimental Lung Research. 2015;41:415-21.

23. Stenlo M, Hyllén S, Silva IAN, Bölükbas DA, Pierre L, Hallgren $\mathrm{O}$, et al. Increased particle flow rate from airways precedes clinical signs of ARDS in a porcine model of LPS-induced acute lung injury. American journal of physiology Lung cellular and molecular physiology. 2020;318:L510-7.

24. Schmidhammer R, Wassermann E, Germann P, Redl H, Ullrich $R$. Infusion of increasing doses of endotoxin induces progressive acute lung injury but prevents early pulmonary hypertension in pigs. Shock. 2006;25:389-94.

25. Zadeh JK, Ruemmler R, Hartmann EK, Ziebart A, Ludwig M, Patzak A, et al. Responses of retinal arterioles and ciliary arteries in pigs with acute respiratory distress syndrome (ARDS). Experimental Eye Research. 2019;184:152-61.
26. Grasselli G, Zangrillo A, Zanella A, Antonelli M, Cabrini L, Castelli A, Cereda D, Coluccello A, Foti G, Fumagalli R, Iotti G, Latronico N, Lorini L, Merler S, Natalini G, Piatti A, Ranieri MV, Scandroglio AM, Storti E, Cecconi M, Pesenti A. Baseline characteristics and outcomes of 1591 patients infected with SARSCoV-2 admitted to ICUs of the Lombardy Region. Italy. JAMA. 2020;323:1574-81.

27. Han S, Mallampalli RK. The acute respiratory distress syndrome: from mechanism to translation. J Immunol. 2015;194:855-60.

28. Girard TD, Bernard GR. Mechanical ventilation in ARDS: a stateof-the-art review. Chest. 2007;131:921-9.

29. Brower RG, Matthay MA, Morris A, Schoenfeld D, Thompson BT, Wheeler A. Ventilation with lower tidal volumes as compared with traditional tidal volumes for acute lung injury and the acute respiratory distress syndrome. N Engl J Med. 2000;342:1301-8.

30. Robertson TE, Mann HJ, Hyzy R, Rogers A, Douglas I, Waxman AB, Weinert C, Alapat P, Guntupalli KK, BuchmanTG. Multicenter implementation of a consensus-developed, evidencebased, spontaneous breathing trial protocol*. Crit Care Med. 2008;36:2753-62.

31. Massagram W, Hafner N, Lubecke V, Boric-Lubecke O. Tidal volume measurement through non-contact doppler radar with DC reconstruction. IEEE Sens J. 2013;13:3397-404.

Publisher's Note Springer Nature remains neutral with regard to jurisdictional claims in published maps and institutional affiliations. 